\title{
A novel approach to conducting clinical trials in the community setting: utilizing patient-driven platforms and social media to drive web-based patient recruitment
}

Janelle Applequist ${ }^{1 *}$ (D), Cristina Burroughs ${ }^{2}$, Artemio Ramirez $\mathrm{Jr}^{1}$, Peter A. Merkel ${ }^{3}$, Marc E. Rothenberg ${ }^{4}$, Bruce Trapnell ${ }^{4}$, Robert J. Desnick ${ }^{5}$, Mustafa Sahin ${ }^{6}$ and Jeffrey P. Krischer ${ }^{2}$

\begin{abstract}
Background: Participant recruitment for clinical research studies remains a significant challenge for researchers. Novel approaches to recruitment are necessary to ensure that populations are easier to reach. In the context of rare diseases, social media provides a unique opportunity for connecting with patient groups that have representatively lower diagnosis rates when compared with more common diseases or illness. We describe the implementation of designing a patient-centered approach to message design for the purposes of recruiting patients for clinical research studies for rare disease populations.

Methods: Using an iterative research approach, we analyzed our previous experience of using web-based direct-topatient recruitment methods to compare these online strategies with traditional center of excellence recruitment strategies. After choosing six research studies for inclusion in the previous study, in-depth, online interviews $(n=37)$ were conducted with patients represented in each disease category to develop and test recruitment message strategies for social media and a Web-based platform for patients to access study information and pre-screen. Finally, relationships were established with Patient Advocacy Groups representing each rare disease category to ensure further dissemination of recruitment materials via their own social media networks.

Results: Guided by social marketing theory, we created and tested various recruitment message designs. Three key message concepts preferred by patients emerged: (1) infographic; (2) positive emotional messages; and (3) educational information for sharing. A base study website was designed and created based on data from patient interviews. This website includes the option for potential participants to pre-screen and determine their eligibility for the study.

Conclusions: Study participants report wanting to be involved in the design and implementation of recruitment approaches for clinical research studies. The application of the aforementioned methods could aide in the evolution of clinical research practices for the recruitment of both rare and common diseases, where patient-centric approaches can help to create targeted messages designs that participants pre-test and support.
\end{abstract}

Keywords: Patient recruitment, Research recruitment, Clinical research, Web-based recruitment, Social media, Social media recruitment, Patient-centered research, Rare diseases, Social marketing theory

\footnotetext{
* Correspondence: applequist@usf.edu; aramirez2@usf.edu

'Zimmerman School of Advertising and Mass Communications, University of

South Florida, 4202 E. Fowler Ave., CIS 1040, Tampa, FL 33620, USA

Full list of author information is available at the end of the article
}

(c) The Author(s). 2020 Open Access This article is distributed under the terms of the Creative Commons Attribution 4.0 International License (http://creativecommons.org/licenses/by/4.0/), which permits unrestricted use, distribution, and reproduction in any medium, provided you give appropriate credit to the original author(s) and the source, provide a link to the Creative Commons license, and indicate if changes were made. The Creative Commons Public Domain Dedication waiver (http://creativecommons.org/publicdomain/zero/1.0/) applies to the data made available in this article, unless otherwise stated. 


\section{Background}

While medical research continues to provide great benefit to society in terms of advancing knowledge and innovation, achieving target rates for study recruitment and accrual remains a challenge. Inability to reach eligible patients for recruitment ultimately reduces the statistical power of studies, incurs economic costs, and may jeopardize funding [1-8]. In the United Kingdom, for example, research suggests that only $55-56 \%$ of randomized controlled trials achieve their target recruitment rate $[9,10] .$. In the conduct of many clinical research studies, especially for rare diseases, obtaining appropriate numbers of study participants at one single institution is often not feasible, creating a need for the organization of multi-center studies that require specially designed infrastructure. In addition to this model being costly, a major problem with the traditional research model is that the majority of potential study subjects are out of reach to the relatively few, geographically-limited clinical centers involved in the trial [11]. Rare disease populations present a particular challenge in terms of trial recruitment, with $32 \%$ of studies citing lack of patient accrual as the most common reason for trial non-completion [12].

The pharmaceutical industry has positioned itself prominently in the media landscape via its practice of direct-toconsumer advertising of prescription drugs, only allowed in the USA [13]. Such advertising provides the public with knowledge of available treatment options, while simultaneously attracting consumers to particular products [14, 15]. Although investigators are more limited in their recruitment budgets when compared to the pharmaceutical industry, the foundations of direct-to-consumer advertising feature methods that may prove useful in the context of research recruitment. Research has investigated such strategies used to increase recruitment and retention in clinical trials, finding that open trials (rather than blinded, placebo trials), telephone reminders to potential participants to respond to study invitations, information leaflets, and recruitment messages emphasizing scarcity are feasible approaches for improvement $[16,17]$. In particular, the use of the Internet and social media platforms as recruitment tools provide areas for deeper exploration, as these sources provide cost-effective, and sometimes free, access to potential participants. Research has taken notice of this area in attempts to broaden participant reach in an attempt to overcome barriers to enrollment in clinical research studies [18-24].

Previous studies have investigated the use of paid advertisements via Facebook, a singular recruitment effort, but have found mixed results. Some studies have found that paid advertisements via Facebook are financially feasible, offer the ability to attract large numbers of individuals, and provide opportunities for connecting with individuals with specific health conditions [25-27]. Other studies, however, concluded that this type of recruitment may yield few participants, and if participants can be obtained, it is a costly process $[28,29]$.

Obtaining sufficient numbers of participants has continued to be a challenge for clinical research, but remains a significant battle in the context of rare diseases [30, 31]. Under the Rare Diseases Act of 2002, rare diseases are classified as those that affect $<200,000$ persons in the United States [32]. Although each such disease may be rare, there are $>6000$ rare diseases and the total number of people with at least one rare disease is large. Nonetheless, due to the low incidence of these individual diagnoses, the recruitment of sufficient numbers for research studies provides a great challenge.

The Rare Diseases Clinical Research Network (RDCRN) is an innovative international clinical research initiative of the Office of Rare Diseases Research (ORDR) and the National Center for Advancing Translational Sciences (NCAT S) consisting of a network of 21 distinct clinical research consortia. The RDCRN coordinates research studies on more than 200 rare diseases. Centralized coordination is provided by the Data Management and Coordinating Center (DMCC) at the University of South Florida (USF). The DMCC houses all RDCRN data and organizes all protocol activity for more than 100 studies of the 21 rare disease consortia via in-house scalable and customizable electronic data capture systems.

Previously, the Vasculitis Clinical Research Consortium, a member of the RDCRN, has tested the use of web-based direct-to-patient recruitment methods in comparison with tradition multicenter recruitment strategies. In The Assessment of Prednisone in Remission (TAPIR) trial, online recruitment strategies via (Webbased and social media strategies) were tested for comparison with traditional center of excellence recruitment strategies. This clinical trial tested whether patients with granulomatosis with polyangiitis (GPA) had better outcomes after their GPA was well-controlled if they stayed on a dose of $5 \mathrm{mg} /$ day of prednisone or fully came off prednisone [33]. The online recruitment arm of the study utilized a Patient Advocacy Group (PAG) website and social media platforms (Facebook, Twitter, and Google+) to direct potential participants to a public study website. The study website featured study information, inclusion and exclusion criteria, and requirements for participation.

In addition to helping reduce the time and costs of conducting clinical research studies, novel approaches toward online direct-to-patient recruitment could help ensure clinical research questions are answered in a timelier fashion, ultimately bringing therapeutic advances to greater numbers of individuals. The implementation of such methods could also aide in the evolution of clinical research practices for both rare and common diseases. 
The main objective of the current study was to use a reflective, mixed-methods approach, focusing on lessons learned from the TAPIR trial, to design an approach to Web-based, social media recruitment that can be tested across a variety of populations (e.g., rare disease type, age, sex, gender, etc.). This study reports on a comprehensive approach to Web-based, direct-to-patient recruitment.

\section{Methods}

This study aimed to design a means for evaluating whether Web-based and social media platforms can be used effectively to recruit patients with rare disease for clinical research studies. We utilized the consolidated criteria for reporting qualitative research (COREQ) checklist for the framing of this study [34]. This design for Web-based patient recruitment is based on four main methods: (1) an iterative, reflective process to determine which aspects of the TAPIR trial could be used to inform new strategies for development, (2) a comprehensive review of the RDCRN's portfolio of studies to determine a set of various protocols for testing, (3) in-depth, online patient interviews to determine appropriate theoretical framework for message design based on identified communication preferences, and (4) the establishment of support for all recruitment marketing efforts with Patient Advocacy Groups (PAGs) associated with each population of rare disease involved with the protocols under study.

\section{How prior research (TAPIR trial) informed current development strategies}

Previous implementation research has documented the ways in which an iterative and reflective process aiming to draw lessons from previously published studies aids in the design of interventions and frameworks for subsequent testing [35]. A comprehensive review of findings from the TAPIR trial was conducted to identify problems to be addressed in the current study. This approach relied upon a constant comparative method of examining TAPIR's features alongside current context to develop feasible approaches to addressing identified problems.

Results of the TAPIR trial included 49 patients in the traditional clinical center recruitment arm, with 10 in the online recruitment arm [33].. Enrollment goals for each arm was 3.3 participants per month, with actual enrollment rates of 0.4 (online recruitment) and 1.8 (traditional recruitment) participants per month. Social media recruitment efforts utilized for the online-recruitment arm resulted in 16,094 individuals visiting the public TAPIR website over an approximate two-year period. Of these website visits, only 82 individuals $(0.5 \%)$ consented to participate in the trial [27]. Of the 82 individuals that provided consent, only 60 (73\%) completed the registration process by answering the questions sent to them via email. Of this, 47 of 60 individuals (78\%) were eligible to participate in the study based on their self-reported responses. Such significant drop-off, referred to in digital marketing as a bounce rate, from website clicks to registration completion signifies a problem. High bounce rates typically indicate that a website has not been designed to target the visitors it desires.

Additionally, iterative assessment of these findings indicated that the overall workflow (from recruitment to registration) may have involved too many "clicks" and separate tasks for completion (e.g. registration via e-mail questionnaire) for potential participants. The TAPIR trial website utilized an interactive informed consent form for individuals to enroll in the study. Once a participant completed the IC, they were sent an e-mail to verify their e-mail address with a link to a registration form with further questions about their disease. Alternatively, the traditional recruitment approach occurred through the clinical practices of individual research sites.

The public TAPIR website featured 6 individual pages, with users needing to click at least twice to reach the informed consent page. The interactive informed consent document utilized on the website was a traditional consent form, which can be quite lengthy, with large amounts of information for potential participants to digest. This design goes against the "three click rule" of website design, which suggests that users should be able to find all relevant information in three mouse clicks or fewer to avoid leaving users frustrated [36].

Data also revealed that more than $2 / 3$ patients that did access the website did so via a mobile device; however, the public website's content was not optimized for mobile use. In the field of health communication, theory is often cited as a crucial component for inclusion in campaign and message development, yet this step was not addressed in the TAPIR study [37]. The evaluation of advertising measures is more organized and cost effective with theoretically-based approaches, as specific measurable constructs can be easily identified and tested [38]. As such, our implementation experience revealed the need for a theoretical construct for message design, complete with message testing phases that incorporate patient feedback.

The TAPIR trial also required online recruitment arm patients to provide their doctors with a Physician packet to complete for confirmation that they were eligible for the trial. Only 35 out of 47 physicians provided additional information about their patients, signifying a loss of patients at this stage, illustrating the importance of seeking ways to engaging clinicians in the recruitment of patients for research [33].

One of the primary lessons learned from the TAPIR study was the need for future research to investigate the ways in which direct-to-patient recruitment, via the online recruitment approach, may differ across various populations. The 
study only looked at one rare disease population (GPA), which featured mean participant ages of 54.8 years (online recruitment arm) and 55.6 years (traditional recruitment arm). Arguably, this demographic may not be the most appropriate population for consideration of Web-based recruitment techniques, as substantial differences in social media and Internet use by age exist, with only $64 \%$ of U.S. adults ages 50-64 using such platforms [39]. As such, it is possible that the online recruitment arm of the study was not as successful because the trial itself, or the population targeted, were not appropriate for consideration across the Web-based landscape.

Although results of the TAPIR study found that the Web-based online recruitment approach was not as effective as the traditional approach, findings did indicate that web-based social media proved successful in mobilizing a substantial number of individuals to the study website [33]. Iterative assessment of the TAPIR study revealed the importance of incorporating existing partnerships with PAGs into the recruitment process, including patients earlier in the recruitment design process, and creating a more detailed, integrated marketing plan that can track the use of social media recruitment tactics. Given that social media recruitment efforts were also reported as appealing via the qualitative data collected from participants, the RDCRN concluded direct-topatient approach is still highly appealing, with further research needed regarding the implementation of a successful Web-based marketing strategy.

\section{Protocol selection process}

While the TAPIR trial's online recruitment approach featured a mean patient age of 54.8, the current study comprehensively reviewed all studies being led by the RDCRN to determine which studies would cover the broadest range of patient ages, rare disease categories, and study requirements. The enrolling studies embedded herein address important clinical questions regarding rare diseases. The rare disease research community has identified these topics as important areas of unmet need and these studies have the potential to impact clinical practice. The current study provides the foundation upon which we will test the proposed novel methods for recruitment, data collection, and overall conduct of rare disease clinical research.

A total of six research studies from 5 rare disease consortia (see Table 1) were chosen for inclusion in our efforts to design a comprehensive direct-to-patient recruitment approach, chosen according to those studies which provide a diverse array of study designs and target populations. Factors including research study design (placebo-controlled, observational, longitudinal, etc.), target demographic participant population, type of investigational agent or device used in the study (investigational agent as compared to a repurposed one), and the level of participant involvement in the study (in-person visits, daily diaries, etc.) were considered in the selection of research studies to include in this protocol. Accordingly, there is no planned accrual target overall and the target enrollment will be that of the RDCRN Consortium study accepted as a stratum, that is, there is no change to each study's target accrual. Each stratum reflects the design of the accepted Consortia protocols.

\section{Patient interviews}

Designing recruitment messages for patient audiences can be challenging, as inclusion and exclusion criteria can seem complex or overly scientific to lay individuals [34]. Therefore, it is important that such messages are designed not only to be attractive, but also to feature content easily understood by audience members [40]. Prior to deciding on messages to be distributed for recruitment, it is essential that formative research be conducted to understand what target audiences want, will attend to, and can understand [41]. Although arguably not often a step taken prior to participant recruitment initiatives, addressing these factors has been shown to increase the probability of behavior change during mass communication campaigns [42]. To facilitate the design of a direct-to-patient recruitment strategy that would best resonate with patients, we implemented in-depth online interviews to test all message iterations created. Institutional Review Board (IRB) approval was obtained prior to any contact with interview and research subjects.

An interview guide (Supplementary File 5) was developed for this study to determine patient preferences for recruitment content platforms, important facets of message design to consider when working with particular rare disease groups, and best times of day to share content via social media. Participants for online interviews were recruited via posts on the RDCRN Facebook page using convenience sampling. Patients recruited were those that represented each of the rare disease categories included in the six chosen protocols. Following the completion of informed consent, patients were individually interviewed via an online video conferencing system (GoToMeeting). All interviews were recorded and lasted 60-90 min. The first author of this study, a female assistant professor whose Ph.D. training focused on qualitative research methods, conducted all online interviews. The lead author took field notes for each interview conducted. A semi-structured approach was used during interviewing, during which participants were shown existing recruitment posts being used by other clinical researchers and asked to provide their feedback. All interview participants received a $\$ 10$ digital Amazon gift card as compensation for their time.

Between May and September of 2018, 37 individual interviews were conducted with patients across each rare 
Table 1 RDCRN PRISM Protocols

\begin{tabular}{|c|c|c|c|c|c|c|c|}
\hline Protocol & Consortium & $\begin{array}{l}\text { Site } \\
\text { Locations }\end{array}$ & $\begin{array}{l}\text { Target } \\
\text { Accrual }\end{array}$ & Study Type & $\begin{array}{l}\text { Intervention } \\
\text { Type }\end{array}$ & Age & Disease Status \\
\hline $\begin{array}{l}\text { Abatacept (CTLA4-Ig) for the Treatment of } \\
\text { Relapsing, Non-Severe, Granulomatosis with } \\
\text { Polyangiitis (ABROGATE) }\end{array}$ & $\begin{array}{l}\text { Vasculitis Clinical } \\
\text { Research Consortium } \\
\text { (VCRC) }\end{array}$ & $\begin{array}{l}\text { US, } \\
\text { Canada, } \\
\text { UK, } \\
\text { Ireland, } \\
\text { Germany }\end{array}$ & 66 & $\begin{array}{l}\text { Interventional } \\
\text { RCT, Phase III }\end{array}$ & $\begin{array}{l}\text { - Double- } \\
\text { blinded } \\
\text { - Placebo- } \\
\text { controlled } \\
\text { - Investigational } \\
\text { agent }\end{array}$ & $\begin{array}{l}15 \\
\text { years } \\
\text { old } \\
\text { and } \\
\text { up }\end{array}$ & $\begin{array}{l}\text { Mild flare- } \\
\text { active disease } \\
\text { at enrollment }\end{array}$ \\
\hline $\begin{array}{l}\text { A Randomized, Multicenter Study for Isolated } \\
\text { Skin Vasculitis (ARAMIS) }\end{array}$ & $\begin{array}{l}\text { Vasculitis Clinical } \\
\text { Research Consortium } \\
\text { (VCRC) }\end{array}$ & $\begin{array}{l}\text { US, } \\
\text { Canada }\end{array}$ & 90 & $\begin{array}{l}\text { Interventional } \\
\text { sequential } \\
\text { multiple } \\
\text { assignment } \\
\text { RCT }\end{array}$ & $\begin{array}{l}\text { - } 3 \text { standard of } \\
\text { care } \\
\text { medications }\end{array}$ & $\begin{array}{l}18 \\
\text { years } \\
\text { old } \\
\text { and } \\
\text { up }\end{array}$ & $\begin{array}{l}\text { Active disease } \\
\text { at enrollment }\end{array}$ \\
\hline $\begin{array}{l}\text { Longitudinal Evaluation of Autoimmune } \\
\text { Pulmonary Alveolar Proteinosis (LongPAP) }\end{array}$ & $\begin{array}{l}\text { Rare Lung Disease } \\
\text { Consortium (RLDC) }\end{array}$ & US & 100 & $\begin{array}{l}\text { Longitudinal, } \\
\text { Observational }\end{array}$ & - None & $\begin{array}{l}\text { All } \\
\text { ages }\end{array}$ & $\begin{array}{l}\text { Active Disease/ } \\
\text { Remission (no } \\
\text { major disease } \\
\text { activity) }\end{array}$ \\
\hline $\begin{array}{l}\text { Newer Direct-Acting Anti-Viral Agents as Sole } \\
\text { Therapy of Porphyria Cutanea Tarda in Sub- } \\
\text { jects with Chronic Hepatitis C }\end{array}$ & $\begin{array}{l}\text { Porphyrias } \\
\text { Consortium (PC) }\end{array}$ & US & 49 & Interventional & $\begin{array}{l}\text { - Open label } \\
\text { - One arm }\end{array}$ & $\begin{array}{l}18 \\
\text { years } \\
\text { old } \\
\text { and } \\
\text { up }\end{array}$ & $\begin{array}{l}\text { PCT with } \\
\text { chronic } \\
\text { Hepatitis C }\end{array}$ \\
\hline $\begin{array}{l}\text { A Randomized Double-Blind Controlled Trial } \\
\text { of Everolimus in Individuals with PTEN } \\
\text { Mutations }\end{array}$ & $\begin{array}{l}\text { Developmental } \\
\text { Synaptopathies } \\
\text { Consortium (DSC) }\end{array}$ & US & 40 & $\begin{array}{l}\text { Interventional, } \\
\text { Phase } 1 / / 1\end{array}$ & $\begin{array}{l}\cdot \text { Placebo- } \\
\text { controlled } \\
\text { - Investigational } \\
\text { agent }\end{array}$ & $\begin{array}{l}5-45 \\
\text { years } \\
\text { old }\end{array}$ & $\begin{array}{l}\text { Outpatients } \\
\text { with PTEN } \\
\text { genetic } \\
\text { mutation }\end{array}$ \\
\hline $\begin{array}{l}\text { A Prospective, Multicenter Study to Compare } \\
\text { and Validate Endoscopic, Histologic, } \\
\text { Molecular, and Patient-Reported Outcomes } \\
\text { in Pediatric and Adult Patients with Eosino- } \\
\text { philic Esophagitis, Gastritis, and Colitis }\end{array}$ & $\begin{array}{l}\text { Consortium of } \\
\text { Eosinophilic } \\
\text { Gastrointestinal } \\
\text { Disease Researchers } \\
\text { (CEGIR) }\end{array}$ & US & 1050 & Observational & - None & $\begin{array}{l}3 \\
\text { years } \\
\text { old } \\
\text { and } \\
\text { up }\end{array}$ & $\begin{array}{l}\text { Active disease } \\
\text { at enrollment }\end{array}$ \\
\hline
\end{tabular}

disease category. Overall participant demographics are reflected in Table 2, with supplementary files 1, 2, 3 and 4 providing demographics related to specific disease populations. The interviewer explained to participants

Table 2 Overall Patient Characteristics and Demographics

\begin{tabular}{lll}
\hline Sex & $\mathbf{n}$ & $\%$ \\
\hline Female & 30 & 81.1 \\
Male & 7 & 18.9 \\
Total & 37 & 100 \\
Race & $\mathbf{n}$ & $\%$ \\
American Indian or Alaskan Native & 0 & 0 \\
Asian & 0 & 0 \\
Black or African American & 0 & 0 \\
Native Hawaiian or Pacific Islander & 0 & 0 \\
Caucasian or White & 36 & 97.3 \\
Unknown or Not Reported & 1 & 2.74 \\
Total & 37 & 100 \\
Ethnicity & $\mathbf{n}$ & $\%$ \\
Hispanic or Latino & 0 & 0 \\
Not Hispanic or Latino & 34 & 91.9 \\
Unknown or Not Reported & 3 & 8.1 \\
Total & 37 & 100 \\
\hline
\end{tabular}

that the purpose of the study was to help design appropriate recruitment content, with no interviewerinterviewee relationships established prior to study commencement. Messages evaluated throughout patient concept testing phases included organic social media recruitment content, e-mail blasts to be sent via the RDCRN Contact Registry, and Web design options.

All de-identified interview transcripts were coded by first author of this study using NVivo software. In order to enhance the credibility of the data analysis, transcripts were also coded using NVivo's auto-code feature, which served as a way of triangulating the primary coder's results. As interviews continued, saturation of responses revealed the points at which our message designs needed to be produced and further edited to align with patient preferences. As such, the Step Approach to Message Design and Testing (SatMDT) was utilized as a theoretical framework to identify target audiences, design message content, pilot test, and evaluate message content (see Fig. 1) for use in the proposed comprehensive approach to direct-to-patient recruitment [43].

\section{PAG involvement}

A principal component of our strategy relied upon soliciting buy-in and commitment from PAG representatives to share and promote our recruitment content via their 


\begin{tabular}{|c|c|c|c|c|}
\hline \multicolumn{2}{|c|}{$\begin{array}{c}\text { STEP } 1 \\
\text { Pre-existing Individual } \\
\text { Characteristics }\end{array}$} & \multirow{2}{*}{$\begin{array}{c}\text { STEP } 2 \\
\text { Message-related } \\
\text { Characteristics } \\
\text { Focus \& Content }\end{array}$} & \multirow{2}{*}{$\begin{array}{c}\text { STEP } 3 \\
\text { Individual Responses } \\
\text { Emotional \& Cognitive }\end{array}$} & \multirow{2}{*}{$\begin{array}{c}\text { STEP } 4 \\
\text { Message Outcomes } \\
\text { Acceptance \& Rejection }\end{array}$} \\
\hline Identify & Elicit & & & \\
\hline $\begin{array}{c}\text { Gender, age, } \\
\text { etc. (relevant } \\
\text { socio- } \\
\text { demographic } \\
\text { variables) } \\
\text { Extent \& nature } \\
\text { of involvement } \\
\text { with behavior }\end{array}$ & $\begin{array}{l}\text { Salient } \\
\text { beliefs }\end{array}$ & $\begin{array}{l}\text { Focus of message } \\
\text { and } \\
\text { Key content of } \\
\text { message - appeal } \\
\text { type (eg., } \\
\text { emotional, } \\
\text { informative, etc) }\end{array}$ & 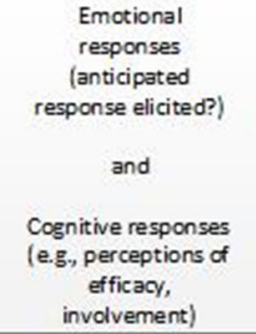 & $\begin{array}{l}\text { Intentions to adopt } \\
\text { message and/or } \\
\text { denial, defensive- } \\
\text { avoidance reactions } \\
\text { Persuasive effects } \\
\text { measured over time }\end{array}$ \\
\hline \multicolumn{2}{|c|}{$\begin{array}{l}\text { Methodology Step } 1 \\
\text { Pilot Work }\end{array}$} & $\begin{array}{l}\text { Methodology Step } 2 \\
\text { Message Exposure }\end{array}$ & $\begin{array}{l}\text { Methodology Step } 3 \\
\text { Concept Testing }\end{array}$ & $\begin{array}{c}\text { Methodology Step } 4 \\
\text { Assessment of Persuasive } \\
\text { Effects }\end{array}$ \\
\hline
\end{tabular}

Fig. 1 The Step Approach to Message Design and Testing (SatMDT). Source: [37]

respective social media platform accounts in order to further promote our efforts. As previously reported by the RDCRN, PAGs act as research partners in ensuring the feasibility and success of various protocols via their collaboration with patient recruitment, support training programs, and study design $[2,44]$.

PAG representatives were asked to provide what they felt would be the most important information to be considered about their respective target population, including the population's geographic characteristics, imagery or messaging that should be utilized or avoided, and most successful recruitment strategies to date. These surveys sought to determine a snapshot of each target population to aid the design team in the creation of recruitment messages. Finally, PAGs were asked to provide detailed information regarding their organization's social media platform preferences, number of followers for each platform, and whether they would be willing to share and promote the RDCRN's direct-to-patient recruitment content online via their own social media accounts. All PAGs agreed to share this content, with the caveat that all posts be pre-approved by PAG representatives and include a statement reiterating that the promotion of the recruitment content does not mean the PAG is endorsing the particular study being advertised.

\section{Results}

Using the previously described methods, we designed an integrated, comprehensive framework for the implementation of social media use in direct-to-patient clinical trial recruitment, titled Protocol for Increasing accrual using Social Media (PRISM). PRISM (see Fig. 2) begins with various recruitment efforts (RDCRN social media posts, PAG sharing of RDCRN social media posts, and use of the RDCRN Patient Contact Registry for e-mail blasts) that lead patients to a PRISM public website that acts as a mechanism for patient-initiated screening and subsequent referral.

\section{How patient feedback and theory informed message design}

Consideration of the recruitment messages shared via social media to lead patients to the public PRISM website was an important first-step. In-depth patient interviews utilized existing research recruitment ads and social media posts from other networks (e.g., pharmaceutical companies, non-profit organizations) to help us better understand what kind of content audiences found most appealing. This enabled the creation of more targeted, organic (nonpaid) content. Three key message concepts emerged from our analysis of the interview data: (1) infographic; (2) positive emotional messages; and (3) educational information for sharing. Creative designs for Facebook and Twitter were created and iteratively modified throughout each round of message testing. Table 3 describes the message concepts developed based on interview responses.

Based on the feedback obtained from our in-depth interviews with patients, it became clear that social marketing theory emerged as the most useful theoretical framework for informing our message designs. Whereas typical marketing campaigns seek to influence purchasing decisions, social marketing campaigns exist to promote socially desirable behaviors that can help others $[45,46]$. Patients often reported that helping to advance science in ways that would help others diagnosed with rare diseases in the future as their main motivator for 


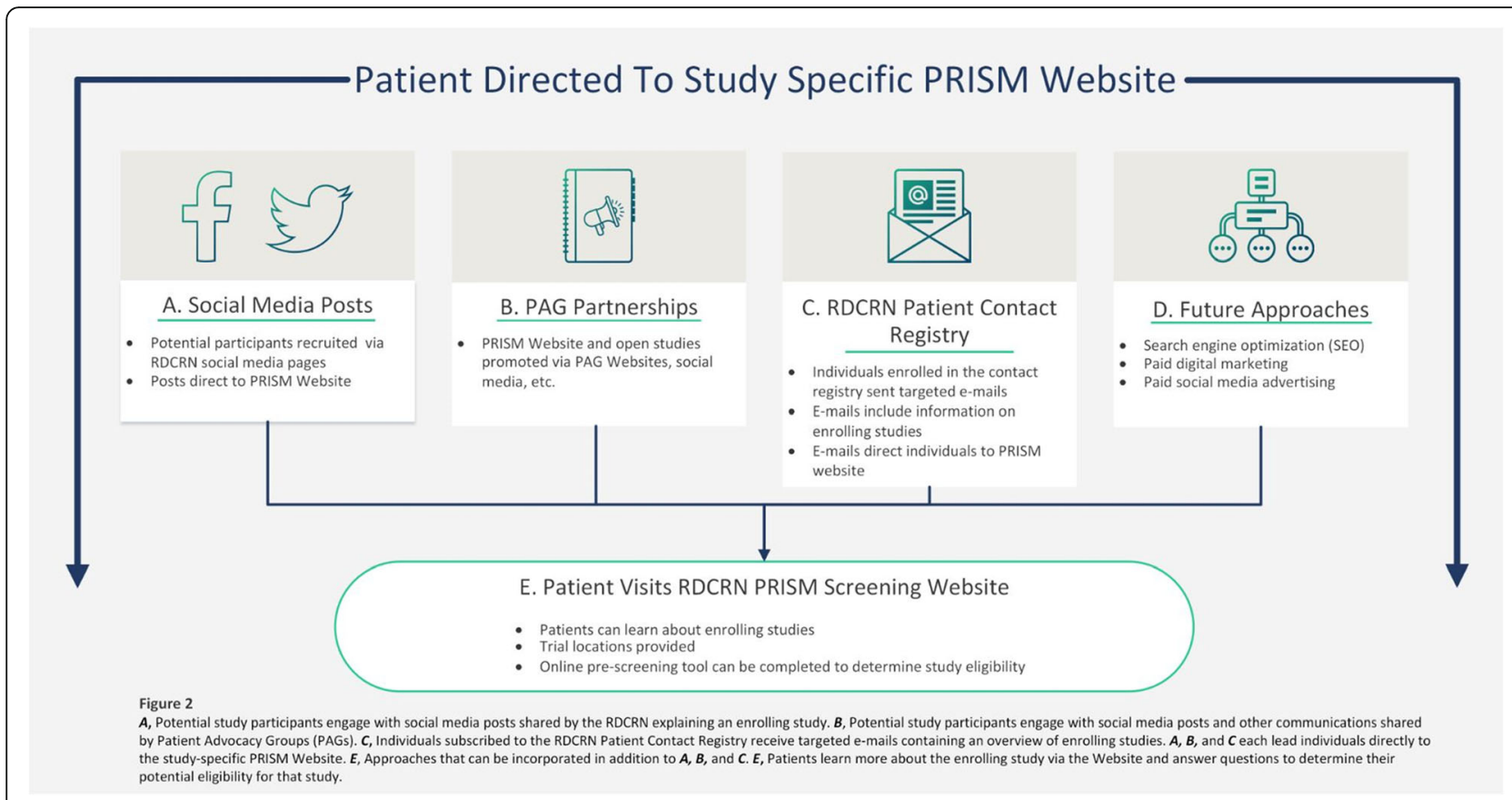

Fig. 2 Comprehensive Approach to Identification and Recruitment of Potential Subjects to PRISM Website

participating in research. As such, our study designed content focused on the motivator of "helping others" to resonate with target audiences. Examples of our content featuring this theme include tag lines such as "we can't do this without you" and "your participation helps others with rare diseases."

Social marketing relies upon a central emphasis on behavior change in coordination with the traditional " 4 P's" of marketing (product, price, place, and promotion) [47]. Social media and Web-based technologies are being used to elicit the behavior change of one choosing to engage with advertisements related to recruitment and ultimately deciding to enroll in a study. In the context of investigators implementing direct-to-patient recruitment via Web-based technologies, the product is the PRISM website. The price for investigators is minimal, in that the implementation of direct-to-patient recruitment techniques requires time and a marketing plan. Place refers to the web-based technologies used to house recruitment materials and the PRISM website. Promotion includes the range of integrated advertising and directto-patient communication content created that are featured in the results section of this manuscript.

\section{PRISM public website}

Clicking on the Facebook and Twitter posts described above lead participants to a study-specific PRISM public website. Our market research informed each step of the design of these pages. During the interview phase, patients often reported being most likely to engage with a study website that was aesthetically pleasing, easy to navigate, and included pertinent study information written in a way

Table 3 PRISM Patient-Preferred Message Concepts

\begin{tabular}{|c|c|c|}
\hline Message Concept & Example Copy & Theme Targeted \\
\hline $\begin{array}{l}\text { Infographic - } \\
\text { Study Criteria }\end{array}$ & $\begin{array}{l}\text { You may qualify if: 1) you have been diagnosed with autoimmune } \\
\text { PAP }{ }^{1} \text {, and: } 2 \text { ) are willing to travel to one of our clinical sites } 3 \\
\text { times over a } 2 \text {-year period. }\end{array}$ & $\begin{array}{l}\text { Preference for high-contrast, graphic designs that } \\
\text { summarize study eligibility criteria in easy-to-understand } \\
\text { terms }\end{array}$ \\
\hline $\begin{array}{l}\text { Photographed } \\
\text { Image - } \\
\text { Emotional Appeal }\end{array}$ & $\begin{array}{l}\text { PTEN hamartoma tumor syndrome is a genetic condition in which } \\
\text { non-cancerous growths, called hamartomas, develop in different } \\
\text { areas of the body. The disease is hereditary, which means it can } \\
\text { be passed from parents to their children. }\end{array}$ & $\begin{array}{l}\text { Patients want to feel emotionally connected to images they } \\
\text { see featured in the recruitment post; use of family or group } \\
\text { images preferred }\end{array}$ \\
\hline Educational Post & $\begin{array}{l}\text { People affected by PCT }{ }^{2} \text { generally experience "photosensitivity," } \\
\text { which causes painful, blistering lesions to develop on sun-exposed } \\
\text { areas of the skin (i.e. the hands and face). } \\
\text { RDCRN is NOW RECRUITING patients with PCT. }\end{array}$ & $\begin{array}{l}\text { Includes more information about the rare disease that } \\
\text { participants can easily share with friends or family to help } \\
\text { them understand their diagnosis }\end{array}$ \\
\hline
\end{tabular}


that they could understand. As these three themes were the primary findings of our interviews, we began designing mock website templates that were shared with patients as interviews continued. We engaged in a constant iterative process where interview feedback was incorporated to edit the website. Once saturation was reached with a Website mockup that ongoing interviews found was well received by patients, the final base Website was built.

The structure and aesthetic of the base study website was designed to be easily adaptable and customizable for specific studies. The PRISM website provides easy-tounderstand, summarized information for patients, such as inclusion/exclusion criteria, study design, and how to participate. Patients felt strongly that the color scheme of the website needed to be professional, yet bright, and they often emphasized their support for the use of the background image (a group of people) because this made them feel they were working together toward a common goal of helping others (see Fig. 3).

The Website workflow (see Fig. 4) begins with patients visiting the Website, where they can learn more information about the study. Individuals are then given the opportunity to screen for their potential eligibility by completing an interactive questionnaire with ten items or less (see Fig. 5). All screening questions were developed based on appropriate health literacy criteria (e.g., using simple language, defining technical terms, using active voice in messaging) and in conjunction with study Principal Investigators (PIs) [48]. If the participant is found to be eligible for a recruiting Consortium clinical study based on self-reported responses, the participant will move to the registration phase. Here, the patient will

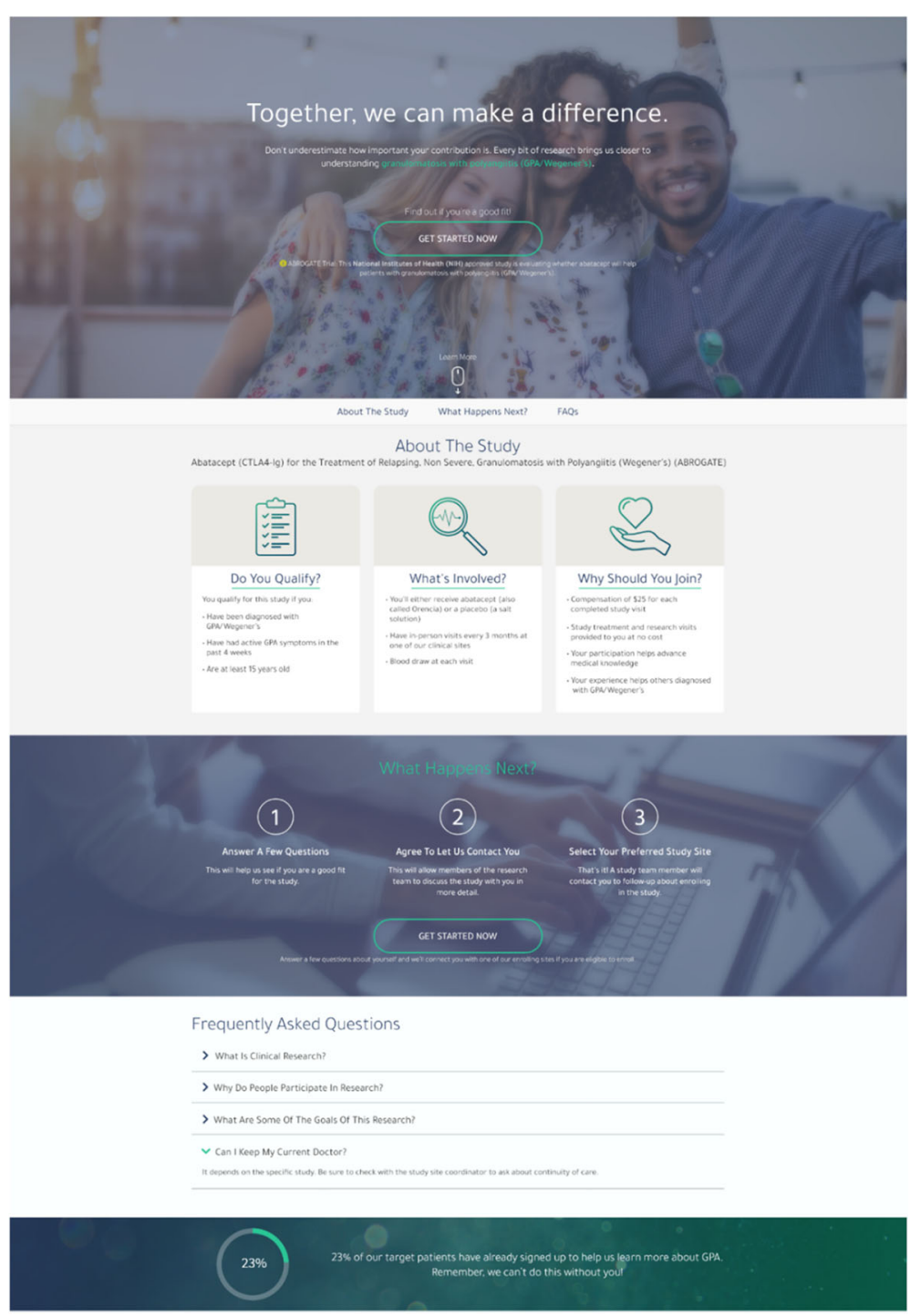

Fig. 3 PRISM Website 


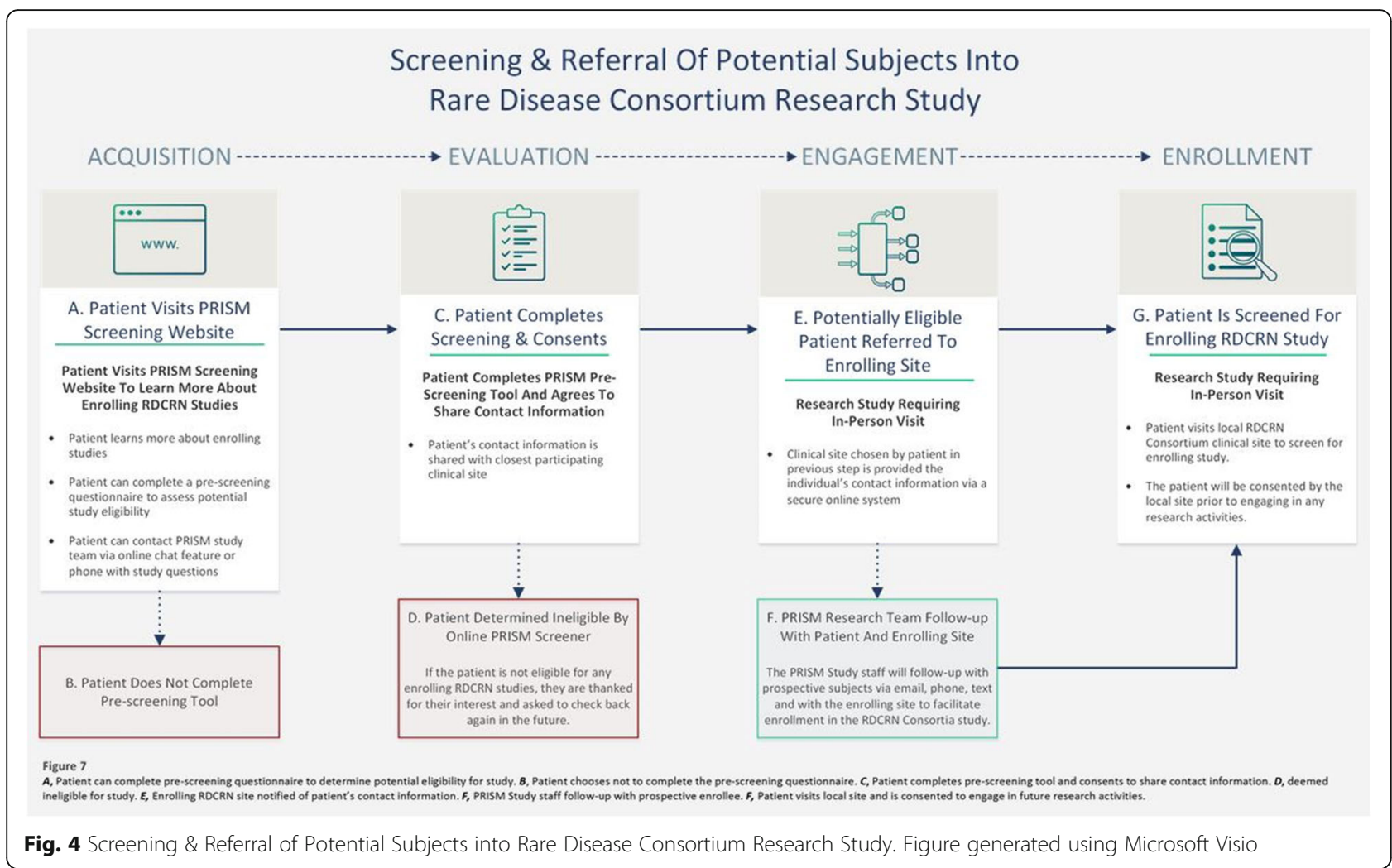

provide their contact information and agree to share this information with PRISM staff and with the enrolling clinical center in their geographic area for studies requiring in-person studies. Patients may also indicate their contact preferences in this step to indicate how study staff may contact them (via phone, email, or text).

For RDCRN Consortia studies that require in-person visits, patients that are deemed potentially eligible based on their self-reported screening responses on the PRISM website will have the option to consent to share their contact information with the RDCRN clinical site of their choice. The PRISM registration process will assist patients in finding the closest enrolling site geographically to them using an embedded Google Maps feature. Each of the rare disease studies chosen for PRISM were selected based on having a varying number of clinical sites and differences in geographic location of sites, allowing us to examine participant willingness to travel for rare disease research once PRISM launches to the public. Based on anecdotal evidence provided by investigators and PAGs, many rare disease patients are willing to travel for their healthcare needs. Geographic availability of site locations for patients is one of the variables our study team plans to explore on acceptance and enrollment of patients in the trial and the subseuqnet response to the PRISM initiative once it is launched. We are interested to examine is a patient's willingness to travel may vary based on features related to the disease population and/or the study intervention. PRISM will further allow us to effectively analyze this conjecture based on the type of study and target disease population.

Patients that do not have a site geographically located near them will have a tool on the interactive website to note, "I do not see a referring site near me" if no enrolling sites are close to their geographic location. These subjects will be referred to the sponsor's study team for follow-up to determine if the patient is able to travel to a participating site or participate remotely, if permitted by the study protocol.

PRISM is a potential participant identification and referral tool. The participant is under the DMCC's IRB for PRISM recruitment and the online pre-screening questionnaire, and must consent to share their contact information with the enrolling RDCRN clinical site of their choice. Once patients have agreed to share their contact information, the patient's contact information and selfreported responses will be provided to the enrolling clinical site via a secure password-protected online members' website maintained by the DMCC. The enrolling clinical site staff will receive an automated notification email alerting site staff that a new patient was referred to their site.

The enrolling clinical site will then contact the referred participant to further assess eligibility for the research study and attempt to bring the participant in for an in-person screening or electronic visit (for studies that do not require in-person visits) if deemed eligible. 


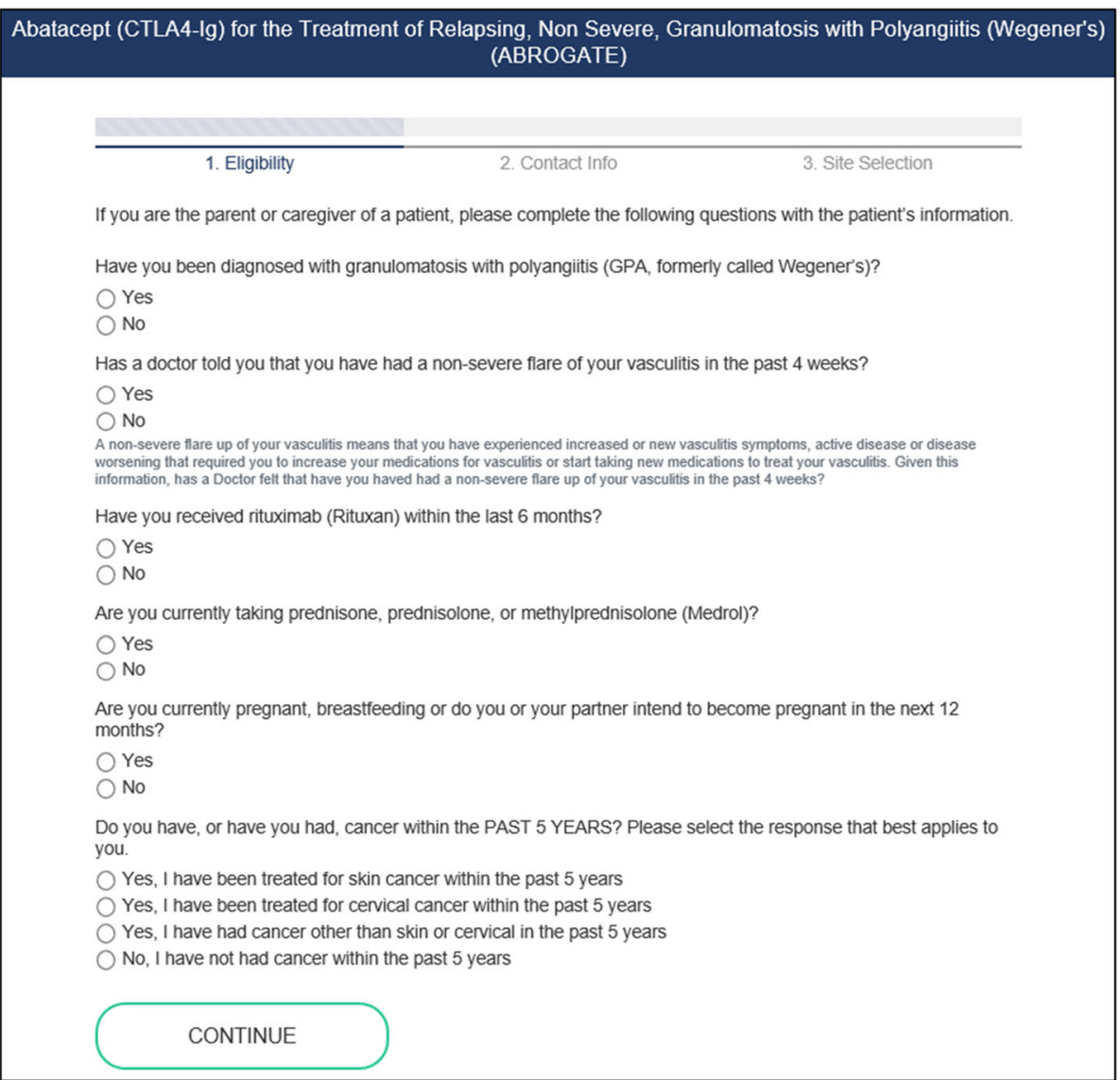

Fig. 5 Online Screener Example

Once the patient has been referred to the enrolling RDCRN Consortium clinical site, the patient will be under the purview of the local site's IRB approved protocol for enrollment in the research study. Thus, the patient must be consented and screened for enrollment in the study as any traditionally recruited subject (for inperson or electronic studies).

For example, a potential study participant with GPA completes the online PRISM screener for the VCRC 5527 Abatacept (CTLA4-Ig) for the Treatment of Relapsing, Non-Severe, Granulomatosis with Polyangiitis (ABROGATE) trial. The online PRISM recruitment via social media, PRISM website self-pre-screening questionnaire and online informed consent form to share their contact information fall under the DMCC's IRB for PRISM-related activities. Once the patient is referred to an enrolling RDCRN clinical site, the local site will need to use their IRB-approved informed consent form for the ABROGATE trial, further discuss the study with the individual, and consent them for the study. The PRISM IRB protocol only covers the recruitment, online prescreening, and referral to enrolling RDCRN clinical sites. Once referred, the patient will follow the local enrolling clinical site's IRB approved enrollment procedures (for both in-person and electronic studies). For studies that do not require in-person visits, the patient would still fall under the study's IRB.

Clinical site personnel will document whether the patients referred to their site via PRISM were deemed eligible by the clinical site staff and if the PRISM participant later enrolls in the clinical study. Enrollment metrics will be regularly evaluated to determine if the online recruitment and referral process requires modifications to improve the screening and referral of potentially eligible research study participants.

Throughout the screening and referral process bidirectional communications via phone, email and text between PRISM study staff and potential subjects will be available to assist with any technical questions the patient may have regarding the online screener and to facilitate enrollment in the RDCRN study. PRISM study staff may also communicate with prospective enrollees and clinical sites throughout this process to ensure the PRISM participant was contacted by the clinical site staff and assist in triaging any questions or concerns the prospective enrollee may have regarding the study enrollment process. 
The funding for ongoing PRISM study staff communication with potential study participants was under the main NIH award for the DMCC at USF. As part of the PRISM initiative, we are evaluating the amount of resources required for participant follow-up (number of emails, phone calls, etc.) and working to automate as many processes as possible to reduce personnel demand. Evaluating the return on investment for telephone or manual follow-up of referred subjects is an important component of the PRISM project to determine efficacy and vitality of the initiative moving forward.

Participant satisfaction with the enrollment process will be solicited following enrollment and regularly monitored by PRISM staff to evaluate and improve the recruitment and referral process. Feedback from RDCRN Consortia clinical site staff and Consortia investigators will be welcomed and incorporated to enhance the screening and referral processes for PRISM.

Data collected for RDCRN study participants identified and enrolled via the PRISM model will be compared to RDCRN study participants recruited and enrolled through other channels to determine if the participant populations recruited through these different avenues differ in demographics, compliance, withdrawal rates or other study metrics.

\section{Discussion}

Evidence suggests that as many as $19 \%$ of clinical trials close without meeting at least $85 \%$ of target accrual rates, signifying the necessity to investigate new methods for implementing novel approaches to research recruitment [49]. This study lead to the design of a comprehensive direct-to-patient recruitment plan to assist in promoting patient opportunities for research, to achieve the goals of the research to improve the health of individuals [50]. Based on lessons learned from the TAPIR trial, PRISM was designed to provide a simple, streamlined process for patients with rare diseases to selfidentify and discover clinical research studies for which they may be eligible to enroll.

PRISM is an online recruitment approach that heavily utilized patient feedback in the production, design, and editing of all recruitment posts and the public Website. The process of comparing and contrasting different protocol types to develop one's own "best practices" for recruitment of specific populations is a logical step for maximizing recruitment efforts. We focused on the rare disease population, but it is clear that there is not a "one size fits all" approach to direct-to-patient recruitment efforts. It is important that any approach to message design taken be done in accordance with market segmentation. The concept of market segmentation (pre-existing individual characteristics) focuses on categories such as gender, average age, and other aspects relating with an identified group when tailoring messages for dissemination [51]. By clearly defining target audiences (e.g., clusters of particular individuals within each rare disease category), researchers can better define message strategies to enhance the likelihood patients will be influenced by the messaging $[18,19,52]$.

To design an appropriate means for evaluating the efficacy of Web-based recruitment strategies, it was considered important that various study populations and trial designs be chosen for inclusion in the new project. Broadening the scope of patient populations allows for the comparison of recruitment strategies based on: the characteristics of the RDCRN study design (e.g. randomized trial, longitudinal study, etc.), demographics of the study population of interest, study requirements of the participant (e.g. online survey, in-person clinical trial, etc.) through metrics such as rate of recruitment, percent eligible, percent drop-out after consent, time from first contact to enrollment, and qualitative feedback from referred patients and RDCRN study staff.

The PRISM website is innovative in that its design and content are patient-focused. Other online research study finder websites (e.g., ClinicalTrials.gov) pull study inclusion and exclusion criteria directly from study protocols, which can be quite confusing for patients to selfnavigate without assistance from healthcare personnel. This process also differs from other online research study finders in that the PRISM provides a communication channel so patients can ask questions to study staff in real time and allow for a personalized study staff member to follow-up with patients to assist with enrollment. This customized enrollment experience seeks to personalize the referral process with potential subjects and assist with eliminating barriers to enrollment in research studies.

The public website designed and used for the TAPIR study included 6 pages, with many user clicks required to reach the informed consent page. The informed consent page itself was lengthy, with qualitative data revealing that patients were often left confused by the questions posed. In response to this, PRISM was designed as a more organized, streamlined process, with primary study information and a link to screen available on the main page and screening questions available on a second page. PRISM adjusted the order in which patients could screen for the study, with screening questions made available to patients before requesting consent to share their contact information. Patients are only asked to consent to share this information if they are deemed to be eligible for the study. In addition, no more than 10 questions are included for patient screening in an effort to keep the process manageable and efficient for patients. Patient information is collected at one time with PRISM, providing an opportunity for clinical sites to more quickly follow-up with patients about enrolling in their respective studies. 
In response to the significant use of mobile devices for accessing the TAPIR study information, PRISM was optimized to be mobile-friendly, with visual icons incorporated to make it easier for patients to read and understand the content being presented via their mobile devices.

In the TAPIR study, patients that did consent tended to drop-off (did not enroll in the study) following their completion of the informed consent form. Part of this may have been because patients were required to verify their e-mail address prior to completing their registration. PRISM, therefore, incorporates a more focused workflow, where patients can read more about the study, screen, and consent to share their contact information all in one step.

Finally, in the TAPIR trial, patients provided their physicians with a packet to complete to confirm their eligibility for the study. Only $74 \%$ of physicians provided this information. Therefore, PRISM removes this barrier. Rather than giving patients the task of having this packet completed, patients are now connected directly with enrolling sites after consenting to provide their contact information.

\section{Limitations}

As in most studies that collect qualitative data, it is difficult to generalize the responses of our rare disease patient population to larger audiences. Individuals with rare diseases may arguably be more enthusiastic and engaged about finding research opportunities, as this population has fewer options in this area when compared to other, more medically-recognized diseases or diagnoses. However, the current study provides important areas for consideration for any area of medicine in the development of approaches to direct-to-patient recruitment. Additionally, exclusively using a Website to find potential participants may preclude individuals who do not have Internet access or use social media from being included in the discovery of study opportunities. As such, it is important that general practitioners and medical staff also advertise and promote the Website's content in order to include all potential participants.

\section{Conclusions}

This study presents one of the first formal applications of previous data to inform the theory based creation of a comprehensive approach to conducting Web-based direct-to-patient recruitment for research. The development of PRISM builds upon the positive findings in the TAPIR trial, seeks to avoid the challenges identified in the approach used for TAPIR, and advances innovative approaches to clinical research by: (1) expanding the use of social media and other online recruitment strategies through the use of novel technologies and marketing campaigns to target populations of patients with rare diseases previously unavailable to researchers [20-24]; (2) identifying and attempting to remove barriers to enrollment and participation in clinical research studies through web-based referral [53]; and (3) demonstrating the feasibility of the proposed methods for clinical research studies in rare disease populations.

\section{Supplementary information}

Supplementary information accompanies this paper at https://doi.org/10. 1186/s12874-020-00926-y.

Additional file 1. Supplementary File 1. Interview Demographics Participant Disease Type. Interview demographics by disease type

Additional file 2. Supplementary File 2. Interview Demographics Participant Sex. Interview demographics by sex

Additional file 3. Supplementary File 3 Interview Demographics Participant Race. Description of data: Interview demographics by race

Additional file 4. Supplementary File 4. Interview Demographics Participant Ethnicity. Interview demographics by ethnicity

Additional file 5. Supplementary File 5 PRISM Patient Participant Interview Guide

\section{Abbreviations}

ABROGATE: Abatacept (CTLA4-Ig) for the Treatment of Relapsing, NonSevere, Granulomatosis with Polyangiitis; Cl: Consortium Investigator; DMCC: Data Management and Coordinating Center; FDA: Food and Drug Administration; GPA: Granulomatosis with polyangiitis; IRB: Institutional Review Board; NCATS: National Center for Advancing Translational Sciences; ORDR: Office of Rare Diseases Research; PAP: Pulmonary Alveolar Proteinosis; PCR: Patient Contact Registry; PCT: Porphyria Cutanea Tarda; PI: Principal Investigator; PM: Project Manager; PRISM: Protocol for Increasing accrual using Social Media; PTEN: Phosphatase and Tensin Homolog;

RCT: Randomized Controlled Trial; RDCRN: Rare Diseases Clinical Research Network; TAPIR: The Assessment of Prednisone in Remission Trial; UK: United Kingdom; US: United States; USF: University of South Florida

\section{Acknowledgements}

The authors greatly appreciate the contributions of the Vasculitis Foundation, the Pulmonary Alveolar Proteinosis (PAP) Foundation, the American

Porphyria Foundation (APF), the PTEN Foundation, the American Partnership for Eosinophilic Disorders (APFED), the Campaign Urging Research for Eosinophilic Disease (CURED), and the Eosinophilic Family Coalition (EFC).

\section{Authors' contributions}

All authors contributed to the conception and design of this work, reviewed drafts of the paper, and read and approved the final version. JA performed all data collection, data analysis, and drafted the paper. CB coordinated aspects of each clinical research study analyzed and assisted in drafting the paper. AR, PAM, MER, BT, RJD, and MS assisted in study design. JK conceived the overall design of the study and oversaw all aspects of the study.

\section{Funding}

This project was supported by the National Heart, Lung, and Blood Institute (NHLBI) (R01HL115041). The Rare Diseases Clinical Research Network (RDCRN) Data Coordinating Center (U01TR001263) is also supported by the Office of Rare Diseases Research (ORDR), via the National Center for Advancing Translational Sciences (NCATS). This project was also supported by the following:

- The Porphyrias Consortium (PC) (U54DK083909) is a part of the RDCRN, an initiative of the ORDR, NCATS. This consortium is funded through a collaboration between NCATS and the National Institute of Diabetes and Digestive Kidney Diseases (NIDDK).

- The Rare Lung Diseases Consortium (RLDC) (U54HL127672) is a part of the RDCRN, an initiative of the ORDR, NCATS. This consortium is funded through a collaboration between the NCATS and the NHLBI. 
- The Developmental Synaptopathies Consortium (DSC) (U54NS092090) is a part of the RDCRN, an initiative of the ORDR, NCATS. This consortium is funded through a collaboration between the NCATS, the National Institute of Neurological Disorders and Stroke (NINDS) and the National Institute of Mental Health (NIMH).

- The Vasculitis Clinical Research Consortium (VCRC) (U54AR057319) is a part of RDCRN, an initiative of the ORDR, NCATS. This consortium is funded through a collaboration between the NCATS and the National Institute of Arthritis and Musculoskeletal and Skin Diseases (NIAMS).

- The Consortium of Eosinophilic Gastrointestinal Disease Researchers (CEGIR) (U54Al117804) is a part of the RDCRN, an initiative of the ORDR, NCATS. This consortium is funded through a collaboration between the NCATS, the National Institute of Allergy and Infectious Diseases (NIAID) and the NIDDK.

\section{Availability of data and materials}

The datasets used and/or analyzed during the current study are available from the corresponding author on reasonable request.

\section{Ethics approval and consent to participate}

The institutional review board (IRB) at the University of South Florida approved this study and all procedures on April 30, 2018 (Protocol number 00034181). Electronic informed consent was obtained for all study participants.

\section{Consent for publication}

Not applicable.

\section{Competing interests}

The authors declare that they have no competing interests.

\section{Author details}

'Zimmerman School of Advertising and Mass Communications, University of South Florida, 4202 E. Fowler Ave., CIS 1040, Tampa, FL 33620, USA. ${ }^{2}$ Health Informatics Institute, University of South Florida, 3650 Spectrum Blvd., Suite 100, Tampa, FL 33612, USA. ${ }^{3}$ Rheumatology Division, University of Pennsylvania, 3400 Spruce St., 5 White, Philadelphia, PA 19104, USA. ${ }^{4}$ Department of Internal Medicine, University of Cincinnati College of Medicine, Medical Science Building, 231 Albert Sabin Way, P.O. Box 670515, Cincinnati, OH 45257-0515, USA. ${ }^{5}$ cahn School of Medicine at Mount Sinai, Icahn (East) Building, Floor 14, Room 14-34, 1425 Madison Ave, New York, NY 10029, USA. ${ }^{6}$ F.M. Kirby Neurobiology Center, Boston Children's Hospital, Center for Life Science, Room 14-073, 3 Blackfan Circle, Boston, MA 02115, USA.

\section{Received: 4 November 2019 Accepted: 14 February 2020}

Published online: 13 March 2020

\section{References}

1. Carlisle B, Kimmelman J, Ramsay T, MacKinnon N. Unsuccessful trial accrual and human subjects protections: an empirical analysis of recently closed trials. Clin Trials. 2015;12:77-83.

2. Cheng K, Gupta SK, Kantor S, Kuhl JT, Aceves SS, Bonis PA, et al. Creating a multi-center rare disease consortium - the consortium of Eosinophilic gastrointestinal disease researchers (CEGIR). Transl Sci Rare Dis. 2017;2(3-4): 141-55.

3. Kitterman DR, Cheng SK, Dilts DM, Orwoll ES. The prevalence and economic impact of low-enrolling clinical studies at an academic medical center. Acad Med. 2011;86:1360-6.

4. Glickman SW, McHutchison JG, Peterson ED, Cairns CB, Harrington RA. Califf RM, etal: ethical and scientific implications of the globalization of clinical research. N Engl J Med. 2009;360:816-23.

5. Hanauer SB. Outsourcing clinical trials. Nat Rev Gastroenterol Hepatol. 2009; 6:191.

6. Peters-Lawrence MH, Bell MC, Hsu LL, Osunkwo I, Seaman P, Blackwood M, et al. Clinical trial implementation and recruitment: lessons learned from the early closure of a randomized controlled trial. Contemp Clin Trials. 2012;33: 291-7.

7. Torgerson JS, Arlinger K, Kappi M, Sjostrom L. Principles for enhanced recruitment of subjects in a large clinical trial. Control Clin Trials. 2001;22: $515-25$.
8. Malmqvist E, Juth N, Lynöe N, Helgesson G. Early stopping of clinical trials: charting the ethical terrain. Kennedy Inst Ethics J. 2011;21:51-78.

9. Walters SJ, Anjos BD, Henriques-Cadby I, Bortolami O, et al. Recruitment and retention of participants in randomized controlled trials: a review of trials funded and published by the United Kingdom Health Technology Assessment Programme. BMJ Open. 2017;7(3):e015276.

10. Sully BGO, Julious SA, Nicholl J. A reinvestigation of recruitment to randomized, controlled, multicenter trials: a review of trials funded by two UK funding agencies. Trials. 2013;14:166.

11. Richesson RL, Lee HS, Cuthbertson D, Young K, Krischer JP. An automated communication system in a contact registry for persons with rare diseases: scalable tools for identifying and recruiting clinical research participants. Contemp Clin Trials. 2009:30:55-62.

12. Rees CA, Pica N, Monuteaux MC, Bourgeois FT. Noncompletion and nonpublication of trials studying rare diseases: a cross-sectional analysis. PLoS Med. 2019;16(11):e1002966.

13. Donohue JM, Cevasco M, Rosenthal MB. A decade of direct-to-consumer advertising of prescription drugs. N Engl J Med. 2007;357:673-81.

14. Applequist J. Broadcast pharmaceutical advertising in the United States: primetime pill pushers. Lanham: Lexington; 2016.

15. Applequist J, Ball JG. An updated analysis of direct-to-consumer television advertisements for prescription drugs. Ann Fam Med. 2018;16(3):211-6.

16. Treweek S, Pitkethly M, Cook J, Fraser C, Mitchell E, Sullivan F, et al. Strategies to improve recruitment to randomised trials. Cochrane Database Syst Rev. 2018;22(2):MR000013.

17. Free CJ, Hoile E, Knight R, Robertson S, Devries KM. Do messages of scarcity increase trial recruitment? Contemp Clin Trials. 2011;32(1):36-9.

18. Smith SK, Selig W, Harker M, Roberts JN, Hesterlee S, Leventhal D, et al. Patient engagement practices in clinical research among patient groups, industry, and academia in the United States: a survey. PLoS One. 2015;10: e0140232.

19. Paul J, Seib R, Prescott T. The internet and clinical trials: background, online resources, examples and issues. J Med Internet Res. 2005;7(1):e5.

20. Marks R, Bristol H, Conlon M, Pepine CJ. Enhancing clinical trials on the internet: lessons from INVEST. Clin Cardiol. 2001;24:17-23.

21. Kopcke F, Prokosch HU. Employing computers for the recruitment into clinical trials: a comprehensive systematic review. J Med Internet Res. 2014; 16(7):e161.

22. Williams IC, Rexrode DL. Beyond traditional advertisements: leveraging Facebook's social structures for research recruitment. J Med Internet Res. 2014 Oct;16(10):e243.

23. Glasgow RE, Nelson CC, Kearney KA, Reid R, Ritzwoller DP, Strecher VJ, et al. Reach, engagement, and retention in an internet-based weight loss program in a multi-site randomized controlled trial. J Med Internet Res. 2007;9(2):e11.

24. Bull SS, Vallejos D, Levine D, Ortiz C. Improving recruitment and retention for an online randomized controlled trial: experience from the Youthnet study. AIDS Care. 2008;20:887-93.

25. Morgan AJ, Jorm AF, Mackinnon AJ. Internet-based recruitment to a depression prevention intervention: lessons from the mood memos study. J Med Internet Res. 2013;15(2):e31.

26. Lohse B, Wamboldt P. Purposive Facebook recruitment endows costeffective nutrition education program evaluation. JMIR Res Protoc. 2013; 2(2):e27.

27. Fenner $Y$, Garland SM, Moore EE, Jayasinghe $Y$, Fletcher A, Tabrizi SN, et al. Web-based recruiting for health research using a social networking site: an exploratory study. J Med Internet Res. 2012;14(1):e20.

28. Kapp JM, Peters C, Oliver DP. Research recruitment using Facebook advertising: big potential, big challenges. J Cancer Educ. 2013;28:134-7.

29. Heffner JL, Wyszynski CM, Comstock B, Mercer LD, Bricker J. Overcoming recruitment challenges of web-based intervention for tobacco use: the case of web-based acceptance and commitment therapy for smoking cessation. Addict Behav. 2013;38:2473-6.

30. Hampton T. Rare disease research gets boost. JAMA. 2006;295:2836-8.

31. Aitken L, Gallagher R, Madronio C. Principles of recruitment and retention in clinical trials. Int J Nurs Pract. 2003;9:338-46.

32. Rare Diseases Act of 2002. [Page 116 STAT, 1988] Public Law 107-280. 107 ${ }^{\text {th }}$ Congress, https://www.congress.gov/bill/107th-congress/house-bill/4013.

33. Krischer J, Cronholm PF, Burroughs C, McAlear C, Borchin R, Easley E, et al. Experience with direct-to-patient recruitment for enrollment into a clinical trial in a rare disease: a web-based study. J Med Internet Res. 2017;19(2):e50. 
34. Tong A, Sainsbury P, Craig J. Consolidated criteria for reporting qualitative research (COREQ): a 32-item checklist for interviews and focus groups. I J Qual Health C. 2007;19(6):349-57.

35. English M. Designing a theory-informed, contextually appropriate intervention strategy to improve delivery of paediatric services in Kenyan hospitals. Implement Sci. 2013;8:1-13.

36. Zeldman J. Taking your talent to the web: making the transition from graphic design to web design. San Francisco: New Riders; 2001.

37. Fishbein M. Introduction to the special section: project SAFER: using theory to identify critical targets for HIV prevention interventions. Psychol Health Med. 2001;6:137-8

38. Maibach E, Parrott RL. Designing health messages: approaches from communication theory and public health practice. Thousand Oaks: Sage; 1995.

39. Smith A, Anderson M. Social media use in 2018. Pew Research Center 2018. https:/www.pewinternet.org/2018/03/01/social-media-use-in-2018/. Accessed 30 September 2019.

40. Fischhoff B. Why (cancer) risk communication can be hard. J Natl Cancer Inst Monogr. 1999;25:7-13.

41. Atkin CK, Freimuth VS. Formative evaluation research in campaign design In: Rice RE, Atkin CK, editors. Public communication campaigns. Thousand Oaks, CA: Sage; 2001. p. 125-45.

42. Nowak GJ, Siska MJ. Using research to inform campaign development and message design: examples from the "American responds to AIDS" campaign. In: Maibach E, Parrott RL, editors. Designing health messages: approaches from communication theory and public health practice. Thousand Oaks, CA: Sage; 1995. p. 169-85.

43. Lewis I, Watson B, White KM. The step approach to message design and testing (SatMDT): a conceptual framework to guide the development and evaluation of persuasive health messages. Accid Anal Prev. 2015;97:309-14.

44. Merkel PA, Manion M, Gopal-Srivastava R, Groft S, Jinnah AH, Robertson D, Krischer JP. The partnership of patient advocacy groups and clinical investigators in the rare disease clinical research network. Orphanet J Rare Dis. 2016;11:1-10.

45. Andreasen A. Marketing social change. San Francisco: Jossey-Bass; 1995.

46. Kotler $\mathrm{P}$, Roberto $\mathrm{N}$, Lee N. Social marketing: improving the quality of life. Thousand Oaks: Sage; 2002

47. Luck J, Hagigi F, Parker LE, Yano EM, Rubenstein LV, Kirchner JE. A social marketing approach to implementing evidence-based practice in VHA QUERI: the TIDES depression collaborative care model. Implement Sci. 2009; 4:1-12.

48. Health literacy basics. U.S. Department of Health and Human Services. https://health.gov/communication/literacy/quickguide/factsbasic.htm. Accessed 18 September 2019.

49. Kiefe $\mathrm{Cl}$, Sales A. A state of-the-art conference on implementing evidence in health care: reasons and recommendations. J Gen Intern Med. 2006;21:S6770.

50. Petty RE, Cacioppo JT. The ELM of persuasion. In: Berkowitz S, editor. Advances in experimental social psychology. New York: Academic; 1986. p. 123-205.

51. Quinn V, Meenaghan T, Brannick T. Fear appeals: segmentation is the way to go. Int J Adv. 1992;11:333-66.

52. Rotfeld H. Misplaced marketing commentary: social marketing and myths of appeals to fear. J Consum Market. 1999;16:119-21.

53. Ross S, Grant A, Counsell C, Gillespie W, Russell I, Prescott R. Barriers to participation in randomized controlled trials: a systematic review. J Clin Epidemiol. 1999:52:1143-56.

\section{Publisher's Note}

Springer Nature remains neutral with regard to jurisdictional claims in published maps and institutional affiliations.

Ready to submit your research? Choose BMC and benefit from:

- fast, convenient online submission

- thorough peer review by experienced researchers in your field

- rapid publication on acceptance

- support for research data, including large and complex data types

- gold Open Access which fosters wider collaboration and increased citations

- maximum visibility for your research: over $100 \mathrm{M}$ website views per year

At $\mathrm{BMC}$, research is always in progress.

Learn more biomedcentral.com/submissions 